\title{
Bottleneck-Oriented Order Release with Shifting Bottlenecks: An Assessment by Simulation
}

Matthias Thürer and Mark Stevenson (corresponding author)

\author{
Name: $\quad$ Prof. Matthias Thürer \\ Institution: Jinan University \\ Address: $\quad$ Institute of Physical Internet \\ School of Electrical and Information Engineering \\ Jinan University (Zhuhai Campus) \\ 519070, Zhuhai, PR China \\ E-mail: matthiasthurer@workloadcontrol.com \\ Name: $\quad$ Prof. Mark Stevenson \\ Institution: Lancaster University \\ Address: $\quad$ Department of Management Science \\ Lancaster University Management School \\ Lancaster University \\ LA1 4YX - U.K. \\ E-mail: $\quad$ m.stevenson@lancaster.ac.uk
}

Keywords: Bottleneck Shiftiness; Bottleneck Position; Drum-Buffer-Rope; Workload Control; Constant Work-In-Process; Simulation. 


\title{
Bottleneck-Oriented Order Release with Shifting Bottlenecks: An Assessment by Simulation
}

\begin{abstract}
Bottleneck shiftiness is an important managerial problem that has received significant research attention. The extant literature has shown, for example, that protective capacity reduces the likelihood of the bottleneck shifting. Yet the actual performance impact of a bottleneck shift has been widely neglected. We posit that there are at least two interrelated effects that may impact shop performance: (i) the direct effect of the change in bottleneck position; and, (ii) the indirect effect of the order release method incorrectly identifying the bottleneck (i.e. assuming the bottleneck is Station X when it is actually Station Y). The latter is particularly acute in the context of bottleneck-oriented order release methods such as Drum-Buffer-Rope (DBR) as these release methods use feedback from the (assumed) bottleneck to control release. Using controlled simulation experiments we demonstrate that a bottleneck shift to a station upstream of the assumed bottleneck has a negligible effect on DBR performance while a downstream shift is detrimental to performance. Meanwhile, the distance, i.e. the number of stations between the actual and assumed bottleneck, has a negligible performance impact. These results have important managerial and research implications for DBR and other release methods.
\end{abstract}

Keywords: Bottleneck Shiftiness; Bottleneck Position; Drum-Buffer-Rope; Workload Control; Constant Work-In-Process; Simulation. 


\section{Introduction}

The phenomenon of shifting bottlenecks bedevils managers (Lawrence \& Buss, 1994) and negatively affects shop floor manageability (Craighead et al., 2001). It is consequently an important problem in practice (Stevenson et al., 2011) that has received significant research attention (e.g. Lawrence \& Buss, 1994; Craighead, 2001; Patterson et al., 2002; Fredendall et al., 2010; Fernandes et al., 2014). The extant literature however has mainly focused on how to avoid bottleneck shiftiness; for example, research has demonstrated that an increase in protective capacity (i.e. capacity at non-bottleneck stations) reduces (but does not eradicate) the likelihood of the bottleneck shifting from one station to another. This research focus has been motivated by the implicit assumption that bottleneck shiftiness is directly detrimental to performance. But this assumption has rarely been questioned; and the actual performance impact of bottleneck shiftiness has rarely been evaluated. In this study, we assess the impact of bottleneck shiftiness on order release performance in a make-to-order flow shop with high variability in terms of the occurrence of demand and processing times. This is a common shop type, such as for companies that focus on producing prototypes and making small runs, e.g. of 1 to 4 units, sometimes referred to as "one offs".

We suggest that there are at least two interrelated effects that may impact shop performance when the bottleneck shifts: (i) the direct effect of the change in bottleneck position; and, (ii) the indirect effect of the order release method incorrectly identifying the bottleneck (i.e. assuming the bottleneck is Station X when it is actually Station Y). The latter is particularly acute in the context of bottleneck-oriented order release methods since they use feedback from the bottleneck to control release.

If order release is applied, jobs are not directly released to the shop floor. Instead, they are withheld in a so-called 'backlog' (Spearman et al., 1990) or 'pool' from which they are released in time to meet certain shop performance metrics. Bottleneck-oriented release 
methods subordinate the release of jobs to the output of the bottleneck. The best-known bottleneck-oriented release method is arguably Drum-Buffer-Rope (DBR) - a descriptor of the way in which order release is realized (Simons \& Simpson, 1997). Meanwhile, bottleneck-oriented release methods have also been presented in the context of Workload Control (e.g. Glassey \& Resende, 1988; Enns \& Prongue-Costa, 2002). A unifying element of these different bottleneck-oriented release methods is that they use a measure of the workload at the bottleneck to regulate the input of work. Using the principles of input/output control (e.g. Plossl \& Wight, 1971), these release methods seek to stabilize the workload at the bottleneck by releasing work in accordance with the output rate.

Aligning the input to the bottleneck with the output assumes however that the bottleneck is known and a feedback loop between the bottleneck and the release function has been established. But a shift in the bottleneck may not be reflected in a shift in the feedback loop. The bottleneck shift may be difficult to predict and short-lived; and it may be disruptive or costly to reflect this shift in the design of the feedback loop. In this paper, we present an exploratory study based on controlled simulation experiments that investigates how making an incorrect assumption about the identity and location of the bottleneck affects shop performance under bottleneck-oriented order release control. In doing so, we consider the impact of distance (i.e. the number of stations between the actual and assumed bottleneck), direction (i.e. whether the actual bottleneck is upstream or downstream of the assumed bottleneck), and bottleneck severity (i.e. the difference in average utilization between the actual bottleneck and all other non-bottleneck stations, which also reflects the stability of the actual bottleneck in the stochastic environment considered). It is hoped that our study provides insights to managers concerning when action, e.g. changing the feedback loop, should be taken in the context of shifting bottlenecks. 
The remainder of this paper is organized as follows. In Section 2, we review the literature on bottleneck shiftiness and on bottleneck-oriented order release control to identify the release methods to be considered in our study. Our research problem and research question are then outlined in Section 3. The simulation model used to evaluate performance is then described in Section 4 before the results are presented, discussed, and analyzed in Section 5. Finally, conclusions are drawn in Section 6, where limitations and future research directions are also outlined.

\section{Literature Review}

We first give a short overview of the literature on shifting bottlenecks in Section 2.1. Section 2.2 then discusses the literature related to bottleneck-oriented release methods considered in our study.

\subsection{Shifting Bottlenecks}

In general, there are three main streams of literature on shifting bottlenecks: shifting bottleneck detection, shifting bottleneck avoidance, and assessments of the performance impact of shifting bottlenecks.

A summary and performance comparison of bottleneck detection methods is contained in Betterton \& Silver (2012). Meanwhile, Yu \& Matta (2016) highlighted the importance of data-driven bottleneck detection and outlined a statistical framework. However, this work does not consider the dynamic phenomenon of shifting bottlenecks. A simple method for calculating the likelihood that a station becomes the bottleneck was presented by Lawrence \& Buss (1994) and Roser et al. (2002). Lawrence \& Buss (1994) also introduced a scalar measure of bottleneck shiftiness. Meanwhile, Roser et al. (2003) outlined a shifting bottleneck detection method, where the capacity resources were Automated Guided Vehicles 
(AGVs). Finally, Roser et al. (2014) presented a simple, practical method for visualizing bottleneck shiftiness - the so-called bottleneck walk.

Research on avoiding bottleneck shiftiness has demonstrated that an increase in protective capacity (i.e. capacity at non-bottleneck stations) reduces bottleneck shiftiness and that a higher level of protective capacity leads to shorter flow times (e.g. Lawrence \& Buss, 1994; Craighead et al., 2001). Craighead et al. (2001) further argued that the flow time is not impacted by the position of protective capacity.

Finally, Fry et al. (1987) found that a bottleneck should be positioned at the first, gateway station and that shifting the bottleneck away from this gateway station may actually lead to performance deterioration. Kadipasaoglu et al. (2000) further clarified that if the bottleneck is the first station then there is no upstream station whose variability may starve it; if the bottleneck is moved downstream, then the probability of the bottleneck starving increases due the cumulative effect of upstream variability. This finding - that a bottleneck at the gateway station leads to the best performance - has recently been confirmed by Thürer et al. (2017a) in the context of an order release controlled flow shop. Meanwhile, Thürer et al. (2017b) highlighted that in high-variety flow and job shops with a bottleneck, workload balancing is less important and bottleneck-oriented release methods have the potential to outperform methods that control the workload at each station. However, this assumes that the bottleneckoriented release method accurately identifies the bottleneck. This assumption is questioned in our study. The bottleneck-oriented release methods to be considered in our study will be discussed next.

\subsection{Bottleneck-Oriented Order Release Methods}

The Theory of Constraints (Goldratt \& Cox, 1984) can be considered a powerful production planning and control technique in shops with bottlenecks. For example, Mabin \& Balderstone (2003) reviewed the literature on more than 80 successful implementations of the Theory of 
Constraints, with $80 \%$ of companies reporting improvements in lead time and due date performance. The Theory of Constraints was originally conceived in the 1970s by Eliyahu M. Goldratt as a scheduling algorithm and later developed into a broader production planning and control concept (Simons \& Simpson, 1997; Mabin \& Balderstone, 2003). One of its main elements is Optimized Production Technology (OPT), a scheduling (or release) mechanism that controls (or subordinates) the release of jobs to the system in accordance with the bottleneck (or constraint). This OPT release mechanism is now more commonly known as Drum-Buffer-Rope (DBR) - a descriptor for the way in which order release is realized (Simons \& Simpson, 1997). A DBR system is depicted in Figure 1 for a single bottleneck station. Its essential parts can be described as follows:

- Drum: This is the constraint (e.g. the bottleneck station, the market) and its schedule.

- Buffer: This is both the constraint buffer (i.e. the buffer before the bottleneck) and the shipping buffer (i.e. the finished goods inventory; see e.g. Watson et al., 2007). Buffers are time (e.g. Radovilsky, 1998; Rahman, 1998; Schragenheim \& Ronen, 1990; Simon \& Simpson, 1997; Chakravorty \& Atwater, 2005; Golmohammadi, 2015) or a timeequivalent amount of work-in-process. Since, in our study, jobs are considered to be delivered immediately after they are completed, the shipping buffer does not exist.

- Rope: This is the communication channel for providing feedback from the drum (bottleneck) to the beginning of the system, i.e. order release. Based on this feedback, order release aligns the input of work with the output rate of the bottleneck. In other words, a maximum limit on the number of jobs released to the bottleneck but not yet completed is established and a job is released whenever the number of jobs is below the prescribed limit (e.g. Ashcroft, 1989; Lambrecht \& Segaert, 1990; Duclos \& Spencer, 1995; Chakravorty \& Atwater, 1996; Chakravorty, 2001; Watson \& Patti, 2008). There are two ropes: Rope 1 determines the schedule at the bottleneck to exploit the constraint 
according to the organization's goal (Schragenheim \& Ronen, 1990); Rope 2 then subordinates the system to the constraint (the bottleneck station).

\section{[Take in Figure 1]}

Another production planning and control approach that has shown promise in shops with bottlenecks is Workload Control (WLC). WLC is a production planning and control concept that has been developed over more than 30 years (Thürer et al., 2011). It has been shown to significantly improve the performance of high-variety shops both through simulation (e.g. Glassey \& Resende, 1988; Land \& Gaalman, 1998; Thürer et al., 2012, 2014) and, on occasions, in practice (e.g. Wiendahl, 1992; Bechte 1994; Hendry et al., 2013; Silva et al., 2015). Although Workload Control has been largely developed in the context of balanced shops, there is some evidence of its potential to improve performance in shops with bottlenecks (e.g. Glassey \& Resende, 1988; Lingayat et al., 1995; Enns \& Prongue-Costa, 2002; Fernandes et al., 2014; Thürer et al., 2017b). For example, Glassey \& Resende (1988) proposed a Starvation Avoidance (SA) methodology. SA releases work whenever the workload of jobs released to the bottleneck but not yet completed falls below a certain predetermined workload limit. This is similar to DBR but controls the workload in time rather than in terms of the number of jobs. Both SA and DBR will be considered in our study. Other WLC release methods that aim to balance the workload across resources are not considered since workload balancing is less important in bottleneck shops (Thürer et al., 2017b).

\section{Problem Definition}

There are two key aspects to bottleneck shiftiness that need to be considered when analyzing the phenomenon: (i) a shift in the actual physical position of the bottleneck (i.e. in the 
layout); and, (ii) a shift in the position of the bottleneck station in the routing of orders. The first aspect, i.e. a shift in the actual physical position of the bottleneck, was considered in Lawrence \& Buss (1994). For example, one time Station C is the bottleneck and the next time Station E is the bottleneck. The second aspect, i.e. a shift in the position of the bottleneck station in the routing of orders, was considered in Hopp \& Spearman (2001; e.g. p. 459). For example, one time Station $\mathrm{C}$ is the bottleneck and the first station in the routing of orders and the next time Station $\mathrm{C}$ continues to be the bottleneck but has moved to being second in the routing sequence. So, while the physical bottleneck station remains the same, its position in the routing of orders changes. One of these aspects often implies the other; however, we make this distinction since the focus of our study - where the order release method is based on an incorrect assumption about the position of the bottleneck - is only relevant in the context of the former aspect. Both SA and DBR control a measure of workload at the bottleneck that requires information to be provided via a feedback loop between the order release point and the bottleneck. When the bottleneck shifts, at best there is a time lag before the feedback loop follows and, at worst, the feedback loop does not shift at all. As a consequence, the order release method makes an incorrect assumption about the position of the bottleneck and takes decisions based on information from a non-bottleneck resource.

In response, this study started by asking: What is the performance impact on bottleneckoriented release methods of making an incorrect assumption about the identity of the bottleneck resource? An exploratory study based on controlled simulation experiments will be used to provide an answer to this question. We will model a pure flow shop in which each job visits all stations in the same routing sequence and in order of increasing station number. This unique shop property allows us to assess the impact of deviation between the assumed and actual bottleneck both in terms of: 
- Direction, i.e. whether the actual bottleneck is upstream or downstream of the assumed bottleneck, and;

- Distance, i.e. the number of stations between the actual and assumed bottleneck.

\section{Simulation Model}

A stylized standard model will be used to avoid interactions that may interfere with our understanding of the experimental factors. While any individual shop in practice will differ in many aspects from our stylized environment, the model used in this study captures the job and shop characteristics of high variety make-to-order flow shops. In other words: shops with high processing time variability and high arrival time variability. The shop and job characteristics modeled in the simulations are first summarized in Section 3.1. How we model the order release methods considered in this study is then outlined in Section 3.2. The priority dispatching rule applied for controlling the progress of orders on the shop floor is described in Section 3.3. Finally, the experimental design is outlined and the measures used to evaluate performance are presented in Section 3.4.

\subsection{Overview of Modeled Shop and Job Characteristics}

A simulation model of a pure flow shop has been implemented in the Python ${ }^{\circledR}$ programming language using the $\mathrm{SimPy}^{\odot}$ simulation module. The shop contains seven stations, where each station is a single resource with constant capacity. There is one bottleneck station, which is varied from Station 1 to Station 7. As in previous research (e.g. Enns \& Prongue-Costa, 2002; Fernandes et al., 2014), non-bottlenecks are created by reducing the corresponding processing times. We experimented with three different levels of bottleneck severity: moderate $=5 \%$; severe $=15 \%$; and very severe $=25 \%$ (processing time reductions). The level of severity also reflects the stability of the actual bottleneck since random shifting will occur in the stochastic production environment considered in this study. The higher the bottleneck 
severity, the less random shifting will occur. An equal adjustment was applied to all nonbottlenecks since the position of protective capacity, i.e. the extra capacity at non-bottleneck resources, is argued to have no effect on flow times (see Craighead et al., 2001). Operation processing times - before adjustment - follow a truncated 2-Erlang distribution with a mean of 1 time unit after truncation and a maximum of 4 time units. The inter-arrival time of jobs follows an exponential distribution with a mean of 1.111 time units, which deliberately results in a utilization level of $90 \%$ at the bottleneck. The average utilization level at nonbottlenecks depends on the degree of bottleneck severity in a given experiment.

Due dates are set exogenously by adding a random allowance factor, uniformly distributed between 28 and 36 time units, to the job entry time. The minimum value will be sufficient to cover a minimum shop floor throughput time corresponding to the maximum processing time (3.8 time units for non-bottleneck operations and 4 time units for the bottleneck operation, which totals 26.8 time units across the seven stations) plus an allowance for the waiting or queuing times. The simulated shop and job characteristics are summarized in Table 1.

\section{[Take in Table 1]}

\subsection{Order Release}

As in previous simulation studies on DBR (e.g. Lambrecht \& Segaert, 1990; Duclos \& Spencer, 1995; Chakravorty \& Atwater, 2005) and Workload Control (e.g. Glassey \& Resende, 1988; Enns \& Prongue-Costa, 2002; Fredendall et al., 2010; Thürer et al. 2017b), it is assumed that all jobs are accepted, materials are available, and all necessary information regarding shop floor routings, processing times, etc. is known. Jobs flow into a pre-shop pool to await release according to two alternative release methods - DBR from the Theory of Constraints and SA (i.e. Starvation Avoidance) from the Workload Control literature. 
- $D B R$ controls the number of jobs released but not yet completed at the (assumed) bottleneck. Whenever a new job arrives at the shop or an operation is completed at the bottleneck, jobs are released until a pre-established buffer limit is reached.

- Starvation Avoidance (SA) controls the workload released but not yet completed at the (assumed) bottleneck.

The station that is assumed to be the bottleneck by the order release method is Station 4, which is the center station, i.e. in the middle of the flow shop. The actual bottleneck is varied from Station 1 to Station 7. Ten buffer limits are applied from 11 to 20 jobs for DBR and from 11 to 20 time units for SA. As a baseline measure, experiments where jobs are released immediately upon arrival at the shop are also included. Finally, jobs are considered for release according to their due date.

\subsection{Shop Floor Dispatching}

The prioritization of jobs on the shop floor is based on the Modified Planned Start Time (MPST) rule, a variant of the Modified Operation Due Date (MODD) rule (e.g. Baker \& Kanet, 1983). The MPST rule prioritizes jobs according to the lowest priority number, which is given by the maximum of the earliest planned finish time and earliest possible finish time, i.e. $\max \left(P S T_{i j}+p_{i j}, t+p_{i j}\right)$ for an operation with processing time $p_{i j}$, where $t$ refers to the time when the dispatching decision is made. The MPST rule shifts between a focus on planned start times (PSTs), to complete jobs on time, and a focus on speeding up jobs - through SPT (Shortest Processing Time) effects - during periods of high load, i.e. when multiple jobs exceed their PST (Land et al., 2015). The planned start time of an operation is determined by successively subtracting a constant allowance for the operation throughput time for each station in the routing of a job from the job's due date. This is similar to the scheduling 
mechanism incorporated in DBR (see, e.g. Chakravorty \& Atwater, 2005). The allowance for the operation throughput time is based on preliminary simulation experiments.

\subsection{Experimental Design and Performance Measures}

The experimental factors are: (i) the seven different positions of the actual bottleneck; (ii) the three different levels of bottleneck severity (moderate, severe and very severe); (iii) the two different release methods (DBR and SA); and (iv) the ten different buffer limit levels for our release methods (from 11 to 20 jobs or time units). A full factorial design was used with 420 cells $(7 * 3 * 2 * 10)$, where each cell was replicated 100 times. Results were collected over 10,000 time units following a warm-up period of 3,000 time units. These parameters allowed us to obtain stable results while keeping the simulation run time to a reasonable level.

The principal performance measures considered in this study are as follows: the lead time - the mean of the completion date minus the pool entry date across jobs; the percentage tardy - the percentage of jobs completed after the due date; and, the mean tardiness $T_{j}=\max \left(0, L_{j}\right)$, with $L_{j}$ being the lateness of job $j$ (i.e. the actual delivery date minus the due date of job $j$ ). In addition to these three main performance measures, we also measure the shop floor throughput time as an instrumental performance variable. While the lead time includes the time that an order waits before release, the shop floor throughput time only measures the time after release to the shop floor.

\section{Results}

Statistical analysis has been conducted by applying ANOVA. ANOVA is here based on a block design with the buffer limit level as the blocking factor, i.e. the different levels of the DBR and SA limits (from 11 to 20) are treated as different systems. A block design allowed the main effect of the buffer limit and both the main and interaction effects of the release method, bottleneck position, and bottleneck severity to be captured. As can be observed from 
Table 2, all main effects, two-way interactions, and three-way interactions were shown to be statistically significant at $\alpha=0.05$.

[Take in Table 2]

The Scheffé multiple-comparison procedure was used to further prove the significance of the differences between the outcomes of our two release methods. Test results, as given in Table 3, suggest that SA outperforms DBR in terms of percentage tardy but that SA is outperformed by DBR in terms of all other performance measures considered in this study. Detailed performance results are presented next in Section 5.1 before a discussion of results is given in Section 5.2.

[Take in Table 3]

\subsection{Performance Assessment}

In this study, we use operating characteristic curves (Olhager \& Persson, 2008) to assess the impact of the bottleneck position. Rather than comparing one specific parameter setting, parameters are varied and the results presented in the form of performance curves. The relative positioning of the different curves (where each curve represents one position of the bottleneck assumed by the order release method) then allows the performance impact to be assessed. In our study, the main parameter determining release method performance is the buffer limit. This parameter is therefore used to create our performance curves. One performance curve for each assumed bottleneck position (from Station 1 to 7 ) is created and presented in Figure 2 and Figure 3 for DBR and SA, respectively.

[Take in Figure 2 \& Figure 3] 
The left-hand starting point of the curves represents the lowest buffer limit. The limit level increases step-wise by moving from left to right, with each data point representing one limit level. Increasing the limit increases the level of work-in-process and, as a result, increases the shop floor throughput time. The results for IMMediate release (IMM), where jobs are released immediately to the shop floor upon arrival and no order release control is applied, are located on the right-hand side since this leads to the highest level of work-in-process on the shop floor.

As somewhat expected, a more severe bottleneck leads to better performance since the total amount of work that has to be processed on the shop floor decreases. Meanwhile, a more severe bottleneck also results in a more pronounced effect when the order release method incorrectly assumes the bottleneck since the effect of random shifting diminishes. This can be observed by moving from Figure $2 \mathrm{a}$ to Figure $2 \mathrm{c}$ and from Figure $3 \mathrm{a}$ to Figure $3 \mathrm{c}$. In terms of direction (i.e. whether the actual bottleneck is upstream or downstream of the assumed bottleneck) and distance (i.e. the number of stations between the actual and the assumed bottleneck) the following can be observed from the results in Figure 2 and Figure 3:

- Direction: In general, we observe a stronger throughput time reduction when the actual bottleneck is upstream (i.e. at Station 1, Station 2, or Station 3) of the assumed bottleneck (Station 4), compared to when the actual bottleneck is downstream of the assumed bottleneck (i.e. at Station 5, Station 6, or Station 7). If the assumed bottleneck is downstream then the actual bottleneck is contained in the control loop and stronger control can be exercised. In fact, for DBR (Figure 2), the performance difference between controlling the actual bottleneck (i.e. when the bottleneck is Station 4 and therefore the assumed and actual bottleneck are the same) and controlling an assumed bottleneck where the actual bottleneck is upstream (i.e. at Station 1, Station 2, or Station 3) appears to be negligible. Meanwhile, for SA (Figure 3), controlling the actual bottleneck significantly 
outperforms all other scenarios. This is because, in contrast to DBR (which controls jobs), SA controls the workload in hours at the station that is identified as the bottleneck.

- Distance: If we only compare the performance curves for stations upstream of the actual bottleneck (Station 4) then the distance between the assumed and actual bottleneck has no clear impact. Performance is however marginally better when distance is at its largest, i.e. when the bottleneck is Station 1. At first, this may appear counter-intuitive, but this is likely to be because Station 1 is the gateway station in our pure flow shop (see also the results in Fry et al., 1987). Meanwhile, if we compare the performance curves for stations downstream of the actual bottleneck then we observe that the further downstream the assumed bottleneck is compared to the actual bottleneck, the worse the mean tardiness performance. This means that here distance does appear to have some impact on performance.

\subsection{Discussion of Results - The Performance Impact of Bottleneck Shiftiness}

Most prior research on bottleneck shiftiness has focused on exploring how protective capacity can be used to reduce shifts in bottleneck position. Research has demonstrated that an increase in protective capacity (i.e. capacity at non-bottleneck stations) reduces bottleneck shiftiness and that a higher level of protective capacity leads to shorter flow times (e.g. Lawrence \& Buss, 1994; Craighead et al., 2001). Craighead et al. (2001) further argued that the flow time is not impacted by the position of protective capacity. Our findings confirm and extend these results. Craighead et al. (2001) controlled the number of jobs in the system. This approach is equivalent to a DBR system (as used in our study) in which the final station is assumed to be the bottleneck. Our results in Figure 2 demonstrate that the impact of the bottleneck shifting to a station that is upstream of the assumed bottleneck is negligible. Thus we extend the findings in Craighead et al. (2001) by arguing that not only does the position of protective capacity have no impact on mean flow times but that the position of the bottleneck 
itself has no or minimal impact. This partially questions the importance of shifting bottlenecks. While it appears to be implicitly assumed in the literature that bottleneck shiftiness has a negative effect on performance, our results argue that the impact on a DBR system is in fact negligible if the shift is to a station upstream of the controlled station; only if the shift is to a station downstream of the controlled station, then bottleneck shiftiness has a direct detrimental impact on performance.

This raises the following question - why not simply control the workload of the whole system by assuming that the last station is the bottleneck regardless of the actual bottleneck position? In this way, the actual bottleneck would always be contained within the control

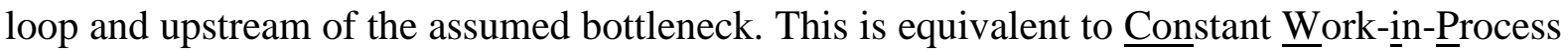
(ConWIP; e.g. Spearman et al., 1990; Hopp \& Spearman, 2001), which releases a new job whenever the number of jobs in the whole system falls below a predetermined limit. It guarantees that the system's actual bottleneck is always contained within the domain of control (Gilland, 2002). Yet, a drawback of ConWIP is that the number of stations contained in a loop should not be too large and the routing of jobs should not differ if the workload and throughput time are to be controlled (Hopp \& Spearman, 2001). The larger the number of stations contained in a ConWIP loop the less the control of the work-in-process in the system (Thürer et al., 2016). Therefore, it may not be advisable to control the whole shop, especially in high-variety contexts. Our study suggests that the design of the DBR/ConWIP loop, e.g. the number of stations incorporated within the loop, should be based on the likelihood of the bottleneck shifting to a station not contained in the loop. This likelihood (and thus the risk of not controlling the bottleneck) has to be traded off against the consequences of the loop containing too many stations. A simple method for calculating the likelihood that a station becomes the bottleneck has been presented by Lawrence \& Buss (1994) and Roser et al. (2002). Meanwhile, Roser et al. (2003) outlined a shifting bottleneck detection method and 
Roser et al. (2014) presented a method for visualizing bottleneck shiftiness - the so-called bottleneck walk.

\section{Conclusions}

Bottleneck shiftiness is an important managerial problem that has received significant research attention. The focus of the extant literature has however mostly been on assessing the impact of protective capacity on bottleneck shiftiness rather than on the actual performance impact of bottleneck shiftiness. We argue that there are at least two interrelated effects that may impact shop performance: (i) the direct effect of the change in bottleneck position; and, (ii) the indirect effect of the order release method incorrectly identifying the bottleneck. The latter effect is particularly acute in the context of bottleneck-oriented release methods. These methods control the input of work to the system based on feedback provided from the assumed bottleneck station. In this study, we therefore asked: What is the performance impact on bottleneck-oriented release methods of making an incorrect assumption about the identity of the bottleneck resource?

Using controlled simulation experiments we have demonstrated that the direction of the shift (i.e. whether the actual bottleneck is upstream or downstream of the assumed bottleneck) influences order release performance. If the bottleneck is upstream of the controlled station then the bottleneck is always contained in the control loop and stronger control can be exercised. In fact, if DBR is used the performance differences become negligible as long as the bottleneck is upstream. Meanwhile, the distance (i.e. the number of station between the actual and the assumed bottleneck) does only influence performance if the actual bottleneck shifts to a downstream station. 


\subsection{Managerial Implications}

The performance outcomes obtained under DBR are hardly affected by a shift in the bottleneck to an upstream station (as seen from the controlled stations). Our results also show that DBR outperforms SA (except in terms of percentage tardy). It is therefore the release method recommended for flow shop environments with shifting bottlenecks in practice. Another important managerial implication that emerges from our results is that management should not 'over-react' to shifts in the bottleneck. If the bottleneck shifts to an upstream station then shifting the feedback loop of the control system may not be necessary. It may even be advisable to orientate control around a station that is downstream of the typical bottleneck rather than around the bottleneck itself. This reduces the risk of a bottleneck shifting 'out of control'. But similar to ConWIP systems, this needs to be carefully considered so as not to incorporate too many stations within the control loop. The more stations that are contained in the control loop, the less control can be exercised. So a trade-off has to be made between the risk of losing control of the bottleneck and the negative performance impact of having too many stations in the control loop. The risk of losing control of the bottleneck can be minimized via the appropriate use of protective capacity; in other words, management can seek to ensure that stations downstream of the controlled station (i.e. assumed bottleneck) have enough protective capacity.

\subsection{Limitations and Future Research}

A major limitation of our study is the reduced environmental setting. We only considered a simple flow shop and neglected factors such as processing time variability, machine break downs, and scrap rates. Our focus on the pure flow shop is justified by the need to clearly identify the direction and distance of a shift in the bottleneck. Meanwhile, the neglect of other environmental factors is due to the need to keep the study to a reasonable level. Future research is however required to assess the impact of these environmental factors on 
performance. Including an extensive set of environmental factors may also shed more light on the relationship between ConWIP and DBR. While previous research has argued that DBR is a better choice than ConWIP, in certain contexts the performance differences between ConWIP and DBR are likely to be negligible. Identifying these contexts and the contingency factors upon which the application of ConWIP vs. DBR is dependent is a major future research direction that emerges from the results of our study.

\section{References}

Ashcroft, S.H., 1989, Applying the principles of optimized production technology in a small manufacturing company, Engineering Costs and Production Economics, 17, 79-88.

Baker, K.R., and Kanet, J.J., 1983, Job shop scheduling with modified operation due-dates, Journal of Operations Management, 4, 1, 11-22.

Bechte, W., 1994, Load-oriented manufacturing control just-in-time production for job shops, Production Planning \& Control, 5, 3, $292-307$.

Betterton, C.E., and Silver, S.J., 2012, Detecting bottlenecks in serial production lines - a focus on interdeparture time variance, International Journal of Production Research, 50, 15, 4158-4174.

Chakravorty, S.S., and Atwater, J.B., 1996, A comparative study of line design approaches for serial production systems, International Journal of Operations \& Production Management, 16, 6, 91-108.

Chakravorty, S.S., 2001, An evaluation of the DBR control mechanism in a job shop environment, OMEGA, 29, 335-342

Chakravorty, S.S., and Atwater, J.B., 2005, The impact of free goods on the performance of drumbuffer-rope scheduling systems, International Journal of Production Economics, 95, 347-357.

Craighead, C.W., Patterson, J.W., and Fredendall, LD., 2001, Protective capacity positioning: impact on manufacturing cell performance, European Journal of Operational Research, 134, 425-438.

Duclos, L.K., and Spencer, M.S., 1995, The impact of a constraint buffer in a flow shop, International Journal of Production Economics, 42, 175-185. 
Enns, S.T., and Prongue Costa, M., 2002, The effectiveness of input control based on aggregate versus bottleneck workloads, Production Planning \& Control, 13, 7, 614 - 624.

Fernandes, N.O., Land, M.J., and Carmo-Silva, S., 2014, Workload control in unbalanced job shops, International Journal of Production Research, 52, 3, 679-690.

Fredendall, L.D., Ojha, D., and Patterson, J.W., 2010, Concerning the theory of workload control, European Journal of Operational Research, 201, 1, 99 - 111.

Fry, T.D., Philipoom, P.R., Leong, G.K., and Smith, A.E., 1987, An Investigation of Bottleneck Position in a Multi-Stage Job Shop, International Journal of Operations \& Production Management, 7, 6, 55-63.

Gilland, W.G., 2002, A simulation study comparing performance of CONWIP and bottleneck-based release rules, Production Planning \& Control, 13, 2, $211-219$.

Glassey, C.R., and Resende, M.G., 1988, Closed-loop job release control for VLSI circuit manufacturing, IEEE Transactions on Semiconductor Manufacturing, 1, 36 - 46.

Goldratt, E.M. and Cox, J., 1984, The Goal: Excellence in Manufacturing, North River Press: New York.

Golmohammadi, D., 2015, A study of scheduling under the theory of constraints, International Journal of Production Economics, 165, 38-50.

Hendry, L.C., Huang, Y., and Stevenson, M., 2013, Workload control: Successful implementation taking a contingency-based view of production planning \& control, International Journal of Operations \& Production Management, 33, 1, 69-103.

Hopp, W.J. and Spearman M.L., Factory Physics: Foundations of Manufacturing Management, Irwin/McGraw-Hill, 2001.

Kadipasaoglu, S.N., Xiang, W., Hurley, S.F., and Khumawala, B.M., 2000, A study on the effect of the extent and location of protective capacity in flow systems, International Journal of Production Economics, 63, 217-228.

Lambrecht, M.R., and Segaert, A., 1990, Buffer stock allocation in serial and assembly type of production lines, International Journal of Operations \& Production Management, 10, 2, 47-61. 
Land, M.J., and Gaalman, G.J.C., 1998, The performance of workload control concepts in job shops: Improving the release method, International Journal of Production Economics, 56-57, 347-364.

Land, M.J., Stevenson, M., Thürer, M., and Gaalman, G.J.C., 2015; Job Shop Control: In Search of the Key to Delivery Improvements, International Journal of Production Economics, 168, 257-266.

Lawrence, S.R. and Buss, A.H., 1994, Shifting production bottlenecks: causes, cures, and conundrums, Production \& Operations Management, 3, $21-37$.

Lingayat, S., Mittenthal, J., and O'Keefe, R., 1995, An order release mechanism for a flexible flow system, International Journal of Production Research, 33, 5, 1241-1256.

Mabin, V.J. and Balderstone, S.J., 2003, The performance of the theory of constraints methodology: analysis and discussion of successful TOC applications, International Journal of Operations \& Production Management, 23, 568-595.

Olhager J., and Persson F., 2008, Using Simulation-Generated Operating Characteristics Curves for Manufacturing Improvement, In: Koch T. (eds) Lean Business Systems and Beyond. IFIP - The International Federation for Information Processing, 257, Springer, Boston, MA

Patterson, J.W., Fredendall, L.D., and Craighead, C.W., 2002, The impact of non-bottleneck variation in a manufacturing cell, Production Planning \& Control, 13, 1, 76 - 85.

Plossl, G.W., and Wight, O.W., 1971, Capacity planning and control, Working paper presented at the APICS International Conference in St.Louis, Missouri.

Radovilsky, Z.D., 1998, A quantitative approach to estimate the size of the time buffer in the theory of constraints, International Journal Production Economics, 55, 113-119.

Rahman, S., 1998, Theory of constraints: a review of the philosophy and its applications, International Journal of Operations \& Production Management, 18, 336-355.

Roser, C., Nakano, M., and Tanaka, M., 2002, Shifting bottleneck detection, Proceedings of the 2002 Winter Simulation Conference, 1079-1086.

Roser, C., Nakano, M., and Tanaka, M., 2003, Comparison of bottleneck detection methods for AGV systems, Proceedings of the 2003 Winter Simulation Conference, 1192-1198.

Roser, C., Lorentzen, K., and Deuse, J., 2014, Reliable Shop Floor Bottleneck Detection for Flow Lines through Process and Inventory Observations, Procedia CIRP, 19, 63-68. 
Schragenheim, E. and Ronen, B., 1990, Drum-buffer-rope shop floor control, Production \& Inventory Management Journal, 31, 18-22.

Silva, C., Stevenson, M., and Thürer, M., 2015, A Case Study of the Successful Implementation of Workload Control: A Practitioner-led Approach, Journal of Manufacturing Technology Management, 26, 2, 280-296.

Simons, J.V. and Simpson, III, W.P., 1997, An exposition of multiple constraint scheduling as implemented in the goal system (formerly disaster), Production \& Operations Management, 6, 322.

Spearman, M.L., Woodruff, D.L., and Hopp, W.J., 1990, CONWIP: a pull alternative to kanban, International Journal of Production Research, 28, 5, 879-894.

Stevenson, M., Huang, Y., Hendry L.C., and Soepenberg, E., 2011, The theory \& practice of workload control: A research agenda \& implementation strategy, International Journal of Production Economics, 131, 2, 689 - 700.

Thürer, M., Stevenson, M., and Silva, C., 2011, Three Decades of Workload Control Research: A Systematic Review of the Literature, International Journal of Production Research, 49, 23, 69056935.

Thürer, M., Stevenson, M., Silva, C., Land, M.J., and Fredendall, L.D., 2012, Workload control (WLC) and order release: A lean solution for make-to-order companies, Production \& Operations Management, 21, 5, 939-953.

Thürer, M., Stevenson, M., Silva, C., Land, M.J., Fredendall, L.D., and Melnyk, S.A., 2014, Lean control for make-to-order companies: Integrating customer enquiry management and order release, Production \& Operations Management, 23, 3, 463-476.

Thürer, M., Stevenson, M., and Protzman, C.W., 2016, Card-based Control Systems for a Lean Work Design: The Fundamentals of Kanban, ConWIP, POLCA and COBACABANA, Productivity Press, ISBN-13: 9781498746946

Thürer, M., Qu, T., Stevenson, M., Li, C.D., and Huang, G.Q., 2017a, Deconstructing Bottleneck Shiftiness: The Impact of the Bottleneck Position in an Order Release controlled Pure Flow Shop, Production Planning \& Control, 28, 15, 1223-1235. 
Thürer, M., Stevenson, M., Silva, C., and Qu, T.; 2017b; Drum-Buffer-Rope and Workload Control in High Variety Flow and Job Shops with Bottlenecks: An Assessment by Simulation; International Journal of Production Economics; 188, 116-127

Watson, K.J., and Patti, A, 2008, A comparison of JIT and TOC buffering philosophies on system performance with unplanned machine downtime, International Journal of Production Research, 46, 7, 1869-1885.

Watson, K.J., Blackstone, J.H., and Gardiner, S.C., 2007, The evolution of a management philosophy: The theory of constraints, Journal of Operations Management, 25, 387-402.

Wiendahl, H.P., Gläßner, J., and Petermann, D., 1992, Application of load-oriented manufacturing control in industry, Production Planning \& Control, 3, 2, $118-129$.

Yu, C., and Matta, A., 2016, A statistical framework of data-driven bottleneck identification in manufacturing systems, International Journal of Production Research, 54, 21, 6317-6332. 
Table 1: Summary of Simulated Shop and Job Characteristics

\begin{tabular}{|c|c|c|}
\hline 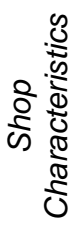 & $\begin{array}{r}\text { Routing Variability } \\
\text { Routing Direction } \\
\text { No. of Stations } \\
\text { Interchange-ability of Stations } \\
\text { Station Capacities }\end{array}$ & $\begin{array}{l}\text { Fixed sequence; no-re-entrant flows } \\
\text { Directed routing (Pure flow shop: PFS) } \\
7 \\
\text { No interchange-ability } \\
\text { All equal }\end{array}$ \\
\hline 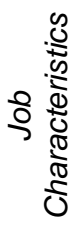 & $\begin{array}{r}\text { No. of Operations per Job } \\
\text { Operation Processing Times (bottleneck) } \\
\text { Operation Processing Times (non-bottleneck) } \\
\text { Due Date Determination Procedure } \\
\text { Inter-Arrival Times }\end{array}$ & $\begin{array}{l}7 \\
\text { Truncated 2-Erlang; }(\operatorname{mean}=1 ; \max =4) \\
\text { Truncated 2-Erlang }(\text { mean }=1 ; \max =4) \text { times } 0.95 \\
\text { (moderate), } 0.85 \text { (severe) and } 0.75(\text { very severe }) \\
\text { Due Date = Entry Time }+d ; d U \sim[28,36] \\
\text { Exp. Distribution; mean }=1.111\end{array}$ \\
\hline
\end{tabular}


Table 2: ANOVA Results

\begin{tabular}{|c|c|c|c|c|c|c|}
\hline & Source of Variance & $\begin{array}{l}\text { Sum of } \\
\text { Squares }\end{array}$ & $\mathrm{df}^{1}$ & Mean Squares & F-Ratio & $\mathrm{p}$-Value \\
\hline \multirow{9}{*}{ Lead Time } & Buffer Limit & 6036.330 & 9 & 670.703 & 564.230 & 0.000 \\
\hline & Release Method (RM) & 302.241 & 1 & 302.241 & 254.260 & 0.000 \\
\hline & Bottleneck Position (BP) & 811.620 & 6 & 135.270 & 113.800 & 0.000 \\
\hline & Bottleneck Severity (BS) & 176001.020 & 2 & 88000.509 & 74029.930 & 0.000 \\
\hline & $\mathrm{RM} \times \mathrm{BP}$ & 32.264 & 6 & 5.377 & 4.520 & 0.000 \\
\hline & $\mathrm{RM} \times \mathrm{BS}$ & 544074.720 & 2 & 272037.360 & 230000.000 & 0.000 \\
\hline & $\mathrm{BP} \times \mathrm{BS}$ & 214.343 & 12 & 17.862 & 15.030 & 0.000 \\
\hline & $\mathrm{RM} \times \mathrm{BP} \times \mathrm{BS}$ & 376.292 & 12 & 31.358 & 26.380 & 0.000 \\
\hline & Error & 49865.420 & 41949 & 1.189 & & \\
\hline \multirow{9}{*}{$\begin{array}{l}\text { Percentage } \\
\text { Tardy }\end{array}$} & Buffer Limit & 3.482 & 9 & 0.387 & 1200.200 & 0.000 \\
\hline & Release Method (RM) & 0.049 & 1 & 0.049 & 151.840 & 0.000 \\
\hline & Bottleneck Position (BP) & 0.587 & 6 & 0.098 & 303.470 & 0.000 \\
\hline & Bottleneck Severity (BS) & 17.621 & 2 & 8.810 & 27331.920 & 0.000 \\
\hline & $\mathrm{RM} \times \mathrm{BP}$ & 0.005 & 6 & 0.001 & 2.380 & 0.027 \\
\hline & $\mathrm{RM} \times \mathrm{BS}$ & 48.113 & 2 & 24.057 & 74628.970 & 0.000 \\
\hline & $\mathrm{BP} \times \mathrm{BS}$ & 0.151 & 12 & 0.013 & 38.940 & 0.000 \\
\hline & $\mathrm{RM} \times \mathrm{BP} \times \mathrm{BS}$ & 0.135 & 12 & 0.011 & 35.010 & 0.000 \\
\hline & Error & 13.522 & 41949 & 0.000 & & \\
\hline \multirow{9}{*}{$\begin{array}{l}\text { Mean } \\
\text { Tardiness }\end{array}$} & Buffer Limit & 1812.267 & 9 & 201.363 & 559.970 & 0.000 \\
\hline & Release Method (RM) & 276.202 & 1 & 276.202 & 768.090 & 0.000 \\
\hline & Bottleneck Position (BP) & 119.817 & 6 & 19.969 & 55.530 & 0.000 \\
\hline & Bottleneck Severity (BS) & 5263.377 & 2 & 2631.688 & 7318.490 & 0.000 \\
\hline & $\mathrm{RM} \times \mathrm{BP}$ & 27.319 & 6 & 4.553 & 12.660 & 0.000 \\
\hline & $\mathrm{RM} \times \mathrm{BS}$ & 18822.390 & 2 & 9411.195 & 26171.710 & 0.000 \\
\hline & $\mathrm{BP} \times \mathrm{BS}$ & 62.454 & 12 & 5.204 & 14.470 & 0.000 \\
\hline & $\mathrm{RM} \times \mathrm{BP} \times \mathrm{BS}$ & 185.388 & 12 & 15.449 & 42.960 & 0.000 \\
\hline & Error & 15084.618 & 41949 & 0.360 & & \\
\hline \multirow{9}{*}{$\begin{array}{l}\text { Throughput } \\
\text { Time }\end{array}$} & Buffer Limit & 19318.259 & 9 & 2146.473 & 3646.820 & 0.000 \\
\hline & Release Method (RM) & 380.620 & 1 & 380.620 & 646.670 & 0.000 \\
\hline & Bottleneck Position (BP) & 7272.665 & 6 & 1212.111 & 2059.350 & 0.000 \\
\hline & Bottleneck Severity (BS) & 77176.431 & 2 & 38588.215 & 65560.630 & 0.000 \\
\hline & $\mathrm{RM} \times \mathrm{BP}$ & 73.575 & 6 & 12.262 & 20.830 & 0.000 \\
\hline & $\mathrm{RM} \times \mathrm{BS}$ & 333613.270 & 2 & 166806.640 & 280000.000 & 0.000 \\
\hline & $\mathrm{BP} \times \mathrm{BS}$ & 578.545 & 12 & 48.212 & 81.910 & 0.000 \\
\hline & $\mathrm{RM} \times \mathrm{BP} \times \mathrm{BS}$ & 698.055 & 12 & 58.171 & 98.830 & 0.000 \\
\hline & Error & 24690.687 & 41949 & 0.589 & & \\
\hline
\end{tabular}

Table 3: Results for Scheffé Multiple Comparison Procedure: Release Methods

\begin{tabular}{|c|c|c|c|c|c|c|c|c|c|}
\hline \multirow{2}{*}{$\begin{array}{l}\text { Rule } \\
\text { (x) }\end{array}$} & \multirow{2}{*}{$\begin{array}{l}\text { Rule } \\
\text { (y) }\end{array}$} & \multicolumn{2}{|c|}{$\begin{array}{l}\text { Lead } \\
\text { Time }\end{array}$} & \multicolumn{2}{|c|}{$\begin{array}{c}\text { Percentage } \\
\text { Tardy }\end{array}$} & \multicolumn{2}{|c|}{$\begin{array}{c}\text { Mean } \\
\text { Tardiness }\end{array}$} & \multicolumn{2}{|c|}{$\begin{array}{l}\text { Throughput } \\
\text { Time }\end{array}$} \\
\hline & & lower $^{1)}$ & upper & lower & upper & lower & upper & lower & upper \\
\hline & & 0.149 & 0.191 & -0.003 & -0.002 & 0.151 & 0.174 & 0.176 & 0.205 \\
\hline
\end{tabular}

1) $95 \%$ confidence interval; * not significant at $\alpha=0.05$ 


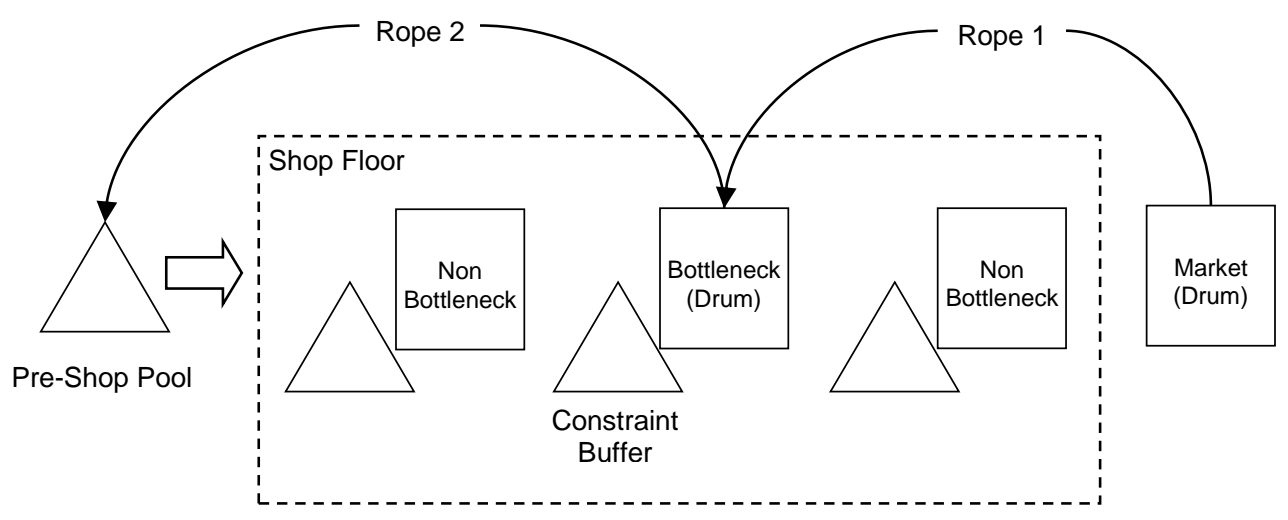

Figure 1: Drum-Buffer-Rope 

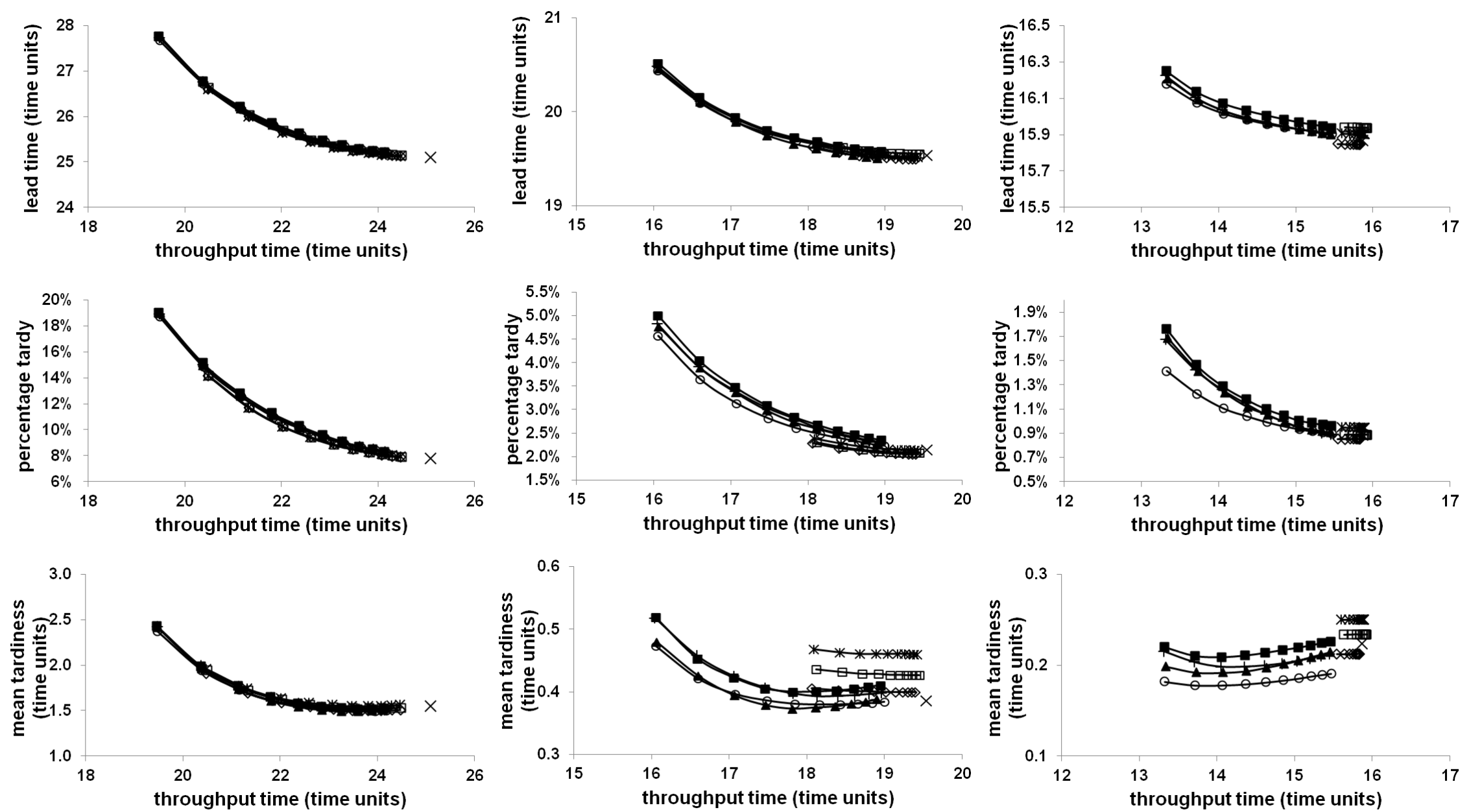

(a)

(b)

Figure 2: Performance Impact of Bottleneck Position under DBR Release: (a) moderate; (b) severe; and, (c) very severe Bottleneck 

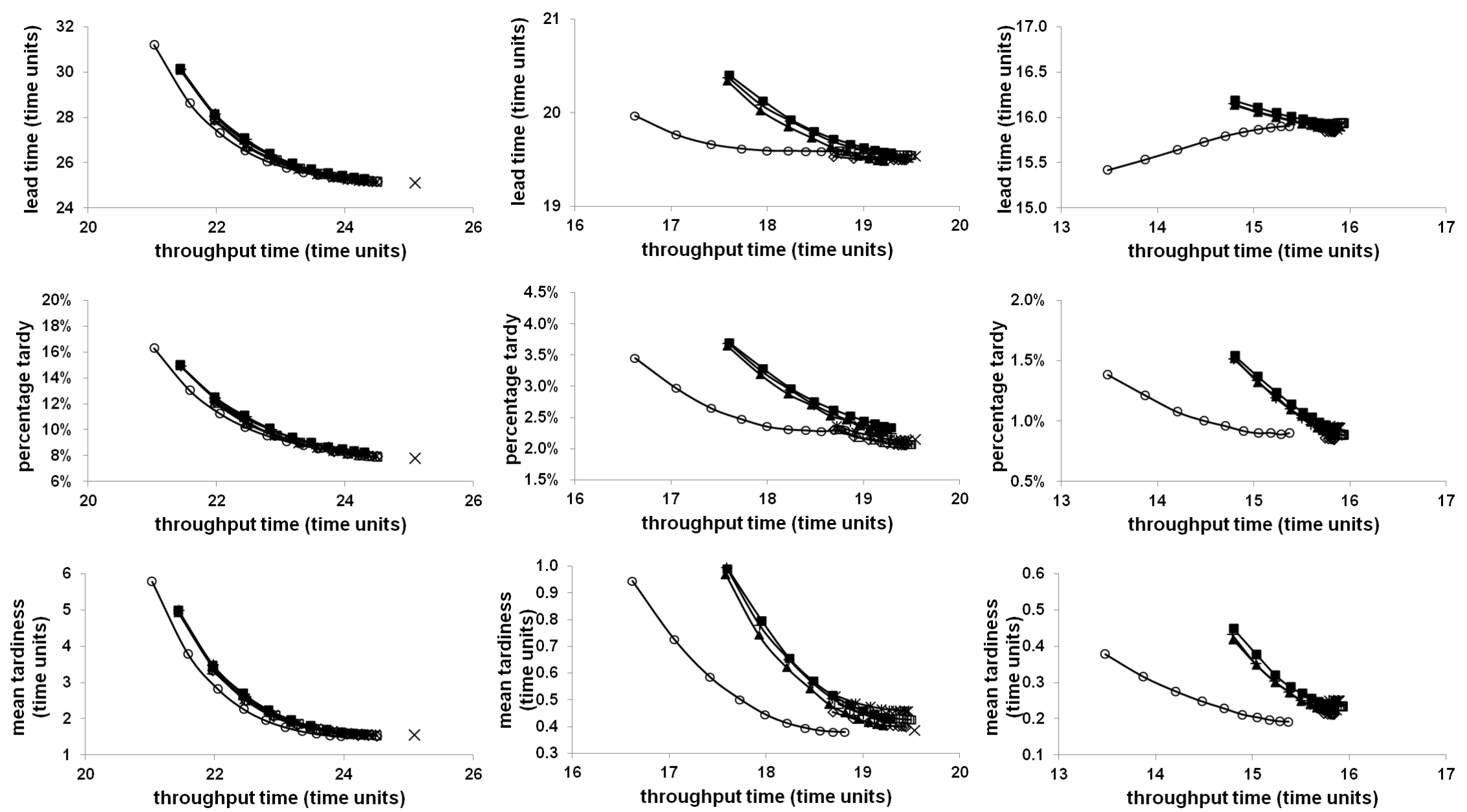

(a)

(b)

(c)

Figure 3: Performance Impact of Bottleneck Position under SA Release: (a) moderate; (b) severe; and, (c) very severe Bottleneck 